\title{
Effects of astaxanthin on the protection of muscle health (Review)
}

\author{
SOK KUAN WONG, SOELAIMAN IMA-NIRWANA and KOK-YONG CHIN \\ Department of Pharmacology, Faculty of Medicine, Universiti Kebangsaan Malaysia, \\ Jalan Ya'acob Latif, Bandar Tun Razak, 56000 Cheras, Kuala Lumpur, Malaysia
}

Received February 3, 2020; Accepted June 17, 2020

DOI: 10.3892/etm.2020.9075

\begin{abstract}
Sarcopenia refers to the involuntary and generalized deterioration of skeletal muscle mass and strength, which may lead to falls, frailty, physical disability, loss of independence, morbidity and mortality. The majority of molecular and
\end{abstract}

Correspondence to: Dr Kok-Yong Chin, Department of Pharmacology, Faculty of Medicine, Universiti Kebangsaan Malaysia, Jalan Ya'acob Latif, Bandar Tun Razak, 56000 Cheras, Kuala Lumpur, Malaysia

E-mail: chinkokyong@ppukm.ukm.edu.my

Abbreviations: 1-RM, one repetition maximum; 4-HNE, 4-hydroxy-2nonenal; 5'-AMPK, 5'-adenosine monophosphate-activated protein kinase; 8-OHdG, 8-hydroxy-2'-deoxyguanosine; $\alpha$-SMA, alpha-smooth muscle actin; Akt, protein kinase B; ANG-1, angiopoietin 1; AOPP, advanced oxidation protein products; ARE, antioxidant responsive elements; AST, aspartate aminotransferase; C/F, capillary-to-fibre; CAF, capillary number per fibre; CAT, catalase; CK, creatine kinase; CSA, cross-sectional area; eNOS, endothelial nitric oxide synthase; FCSA, fibre cross-sectional area; FDA, Food and Drug Administration; Flt-1, FMS-like tyrosine kinase 1; FNDC5, fibronectin type III domain-containing protein 5; FoxO, Forkhead Box O; FoxO3a, Forkhead box class O 3a; FRAP, ferric-reducing activity of plasma; GCLC, glutamate-cysteine ligase catalytic subunit; GCLM, glutamate-cysteine ligase modifier subunit; GPx, glutathione peroxidase; HbAlc, glycated haemoglobin; HIF-1 $\alpha$, hypoxia inducible factor-1 alpha; HMOX-1, heme oxygenase (decycling) 1; HOMA-IR, homeostatic model assessment of insulin resistance; IGF-1, insulin growth factor-1; KDR, kinase insert domain-containing receptor; Keap1, Kelch-like ECH-associated protein 1; MDA, malondialdehyde; MDF, mean dynamic force; MIF, mean isometric force; MVC, maximal voluntary contraction; MPO, myeloperoxidase; mTOR, mammalian target of ripamycin; NEFA, non-esterified fatty acids; NQO-1, $\mathrm{NAD}(\mathrm{P}) \mathrm{H}$ quinone dehydrogenase; Nrf2, nuclear factor erythroid 2-related factor 2; P70S6K, p70 ribosomal protein S6 kinase; PGC-1 $\alpha$, peroxisome proliferator-activated receptor gamma coactivator 1-alpha; PI3K, phosphatidylinositol-3-kinase; RONS, reactive nitrogen species; ROS, reactive oxygen species; SDH, succinate dehydrogenase; SOD, superoxide dismutase; TBARS, thiobarbituric acid-reactive substances; TEAC, trolox-equivalent antioxidant capacity; TGF- $\beta 1$, transforming growth factor-beta 1; Tie-2, tyrosine kinase with Ig and EGF homology domains 2; TUNEL, terminal deoxynucleotidyl transferase dUTP nick end labelling; VEGF, vascular endothelial growth factor

Key words: astaxanthin, mitochondria, muscle atrophy, oxidative stress, sarcopenia cellular changes involved in the degeneration of muscle tissues are mediated by oxidative stress. Therefore, astaxanthin may act as a potential adjunct therapy for sarcopenia owing to its antioxidant activity. The present review examines the effects of astaxanthin on the promotion of skeletal muscle performance and prevention of muscle atrophy and the potential mechanisms underlying these effects. The available evidence till date was retrieved from PubMed and Medline electronic databases. The present review reported the beneficial effects of astaxanthin in preventing muscle degeneration in various animal models of sarcopenia. In humans, the effects of astaxanthin in combination with other antioxidants on muscle health are mixed, wherein positive and negligible effects were reported. Mechanistic studies revealed that astaxanthin promotes muscle health by reducing oxidative stress, myoblast apoptosis and proteolytic pathways while promoting mitochondria regeneration and formation of blood vessels. Thus, astaxanthin is a potential therapeutic agent for sarcopenia but its effects in humans require further validation.

\section{Contents}

1. Introduction

2. Literature search

3. Effects of astaxanthin on skeletal muscle: Evidence from in vivo studies

4. Effects of astaxanthin on skeletal muscle: Evidence from human studies

5. The mechanism of action of astaxanthin

6. Perspectives and conclusion

\section{Introduction}

Sarcopenia is a condition characterized by a progressive reduction in skeletal muscle mass and strength, which affects balance, mobility, overall physical performance and quality of life (1). Risk factors for sarcopenia include increased age, being of the male sex, malnutrition and a sedentary lifestyle (2). Primary sarcopenia is often age-related without apparent underlying causes, whereas secondary sarcopenia is associated with one or more causes (1). Major co-morbidities associated with sarcopenia are obesity, osteoporosis and type 2 diabetes mellitus (3). A meta-analysis of 35 studies showed that the global prevalence of sarcopenia is $10 \%$ in 
men and women, and the prevalence is higher in non-Asian countries than in Asian countries (4). It is estimated that the cost of hospitalisation associated with sarcopenia was approximately $\$ 40.4$ billion USD in the United States, with an average cost of \$260 USD per individual (5). Sarcopenia has also become a topic of interest in recent years, as an increasing proportion of the global population being of advanced age is projected to triple between 2017 and 2050 (6). The development of prophylactic and therapeutic strategies for sarcopenia may therefore become imperative to ensure healthy ageing. It is also noteworthy that there are no US Food and Drug Administration (FDA)-approved drugs for the treatment of sarcopenia (7). Understanding the pathogenesis of sarcopenia will be important in the design of prophylactic and therapeutic strategies for the disease. Oxidative stress, inflammation, impairment of mitochondrial function, increased protein turnover and capillary regression can result in the loss of skeletal muscle mass and ultimately sarcopenia (8-11). Previous studies have demonstrated that the administration of antioxidants reduced the level of oxidative stress during exercise (12) and the level of muscle atrophy (13). These findings suggest a potential role for antioxidants in reversing sarcopenia.

Astaxanthin is a fat-soluble, naturally occurring xanthophyll carotenoid identified in numerous organisms, such as microalgae, crustaceans and fish (such as salmon and trout) (14). Astaxanthin is a powerful antioxidant that effectively scavenges free radicals, quenches singlet oxygen, enhances antioxidant activities and reduces oxidative stress (15). The nutraceutical applications of astaxanthin previously reported include anti-inflammatory (16), anti-cancer (17) and anti-diabetic (18) and it has also been reported to have gastro- (19), hepato- (20), neuro- (21), cardio- (22), ocular- (23) and skin-protective (24) properties.

A recent review summarised the potential application of astaxanthin as a dietary supplement in exercising humans. The author concluded that there was an improvement of exercise metabolism, performance and recovery following astaxanthin supplementation (25). In addition, an in vitro study by Yu et al (26) demonstrated that incubation of the mouse myoblast $\mathrm{C} 2 \mathrm{C} 12$ cell line with astaxanthin $(5 \mu \mathrm{M})$ during heat stress $\left(43^{\circ} \mathrm{C}\right)$ prevented adverse changes to the tubular mitochondrial structure and mitochondrial membrane potential, as well as reactive oxygen species (ROS) production. Thus, astaxanthin may have a potential application in preventing muscle injury and degeneration. In the present review, the health-promoting effects of astaxanthin on the skeletal muscle in animal models and humans are presented. The molecular mechanisms underlying the health benefits of astaxanthin in reversing adverse muscle changes are also highlighted.

\section{Literature search}

Evidence acquisition was conducted between February 1st and February 29th 2020 using PubMed and Medline electronic databases. The key words used to perform the search were 'astaxanthin AND (sarcopenia OR muscle)'. All in vitro, in vivo and human studies detailing the effects of astaxanthin and its underlying mechanisms on muscle health were extracted. A total of 20 related studies are included in the present review.

\section{Effects of astaxanthin on skeletal muscle: Evidence from in vivo studies}

The effects of astaxanthin on skeletal muscle have been explored in vivo (Table I). Recently, Aoi et al (27) compared the effects of three different forms of astaxanthin on endurance performance in 8-week-old ICR mice. Astaxanthin derived from Haematococcus pluvialis (esterified form), synthetic astaxanthin (non-esterified form) or astaxanthin derived from Phaffia rhodozyma (non-esterified form) was provided to the animals in their diet at a dose of $0.02 \%(\mathrm{w} / \mathrm{w})$ for five weeks. The animals were subjected to treadmill exercise with a running speed of $25 \mathrm{~m} / \mathrm{min}$ for the assessment of endurance and their running time to exhaustion was measured. The study indicated that animals fed with astaxanthin from $H$. pluvialis had the longest running time to exhaustion among the experimental groups (27). Long-term effects of astaxanthin supplementation were also evaluated using an exercised animal model. Adult male Wistar rats were administered mineral oil (vehicle) or astaxanthin $(1 \mathrm{mg} / \mathrm{kg})$ five days per week for 45 days. The animals treated with astaxanthin had a higher elapsed time until exhaustion in a forced-swimming activity when compared with exercised animals without treatment (28).

In another study, Kawamura et al (29) investigated the effects of astaxanthin alone, or in combination with other antioxidants ( $\beta$-carotene and resveratrol), on muscle atrophy in 7-week-old male ICR mice. The knee and ankle joints of one hindlimb were fixed with a cast to induce muscle atrophy and removed after three weeks. After cast removal, the animals were fed a basal diet enriched with astaxanthin, $\beta$-carotene, resveratrol or a mixture of the three antioxidants for two weeks. The animals given a basal diet with astaxanthin alone or a mixture of the three antioxidants had significantly higher soleus muscle weight when compared to the normal animals (29).

An animal model of hindlimb unloading was also used to assess the effects of astaxanthin on atrophied soleus muscle. Kanazashi et al (30) performed hindlimb unloading on adult male Wistar rats for 7 days by suspending the tail, to prevent weight bearing of the hindlimb on the floor or contact with the sides of the cage. Astaxanthin was administered orally at $50 \mathrm{mg} / \mathrm{kg}$ twice per day for 7 days. Astaxanthin was demonstrated to prevent the changes caused by hindlimb unloading, indicated by the preserved capillary-to-fibre $(\mathrm{C} / \mathrm{F})$ ratio, capillary number per fibre (CAF), capillary volume and capillary diameter of the treated group. As astaxanthin supplementation alone was beneficial in preventing capillary regression, while exerting minimal impact on muscle mass, the same group of researchers hypothesized that a combination of astaxanthin and intermittent loading would work synergistically on the prevention of muscle atrophy and capillary regression during hindlimb unloading. In the subsequent study, the animals were subjected to hindlimb unloading followed by the release of the suspension device to allow for normal cage activity for one hour daily in darkness. The study duration was extended to two weeks. As expected, the results indicated that intermittent unloading combined with astaxanthin ameliorated both soleus muscle atrophy and capillary regression in the hindlimb unloaded animals (31). A recent study was conducted to 


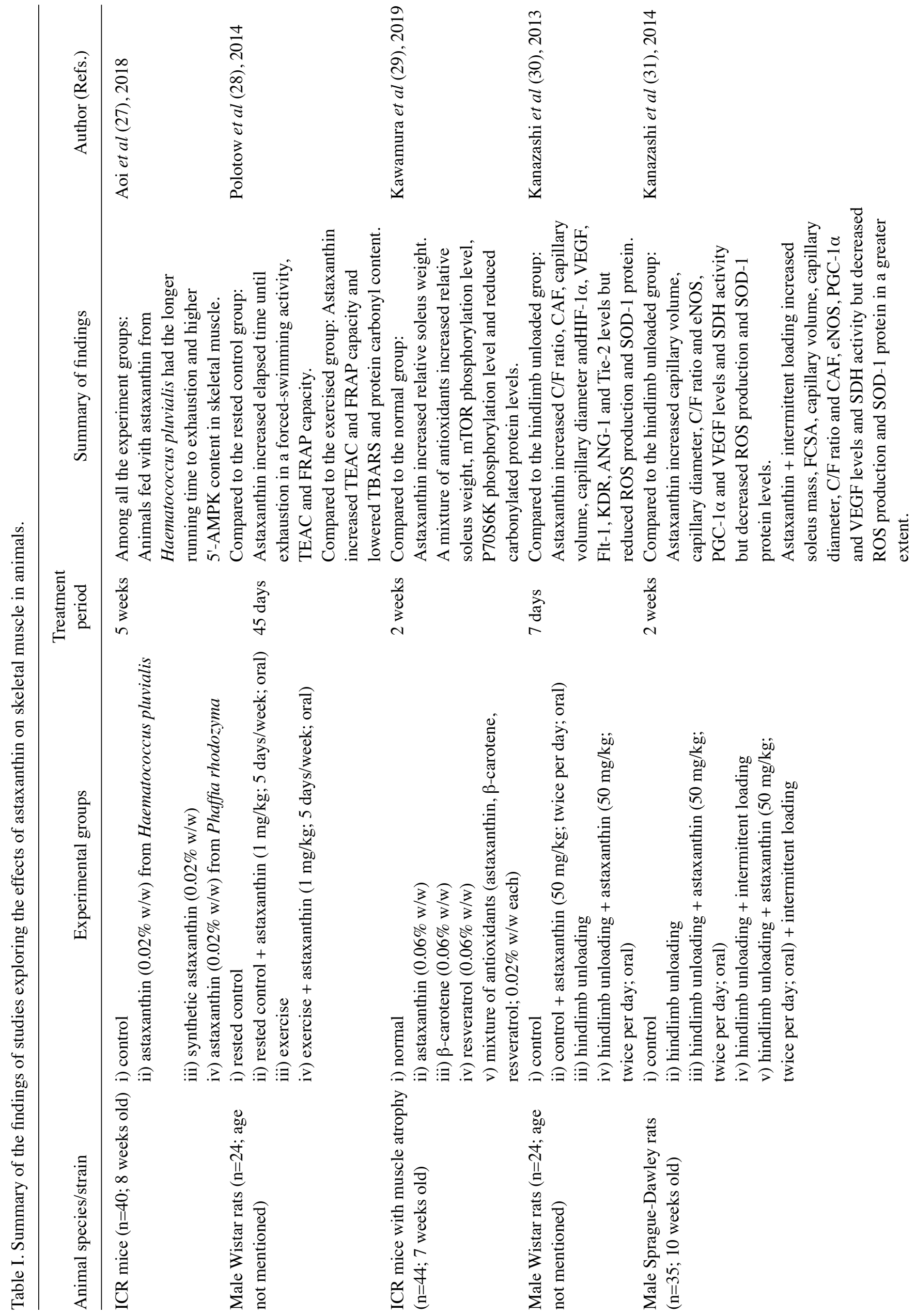




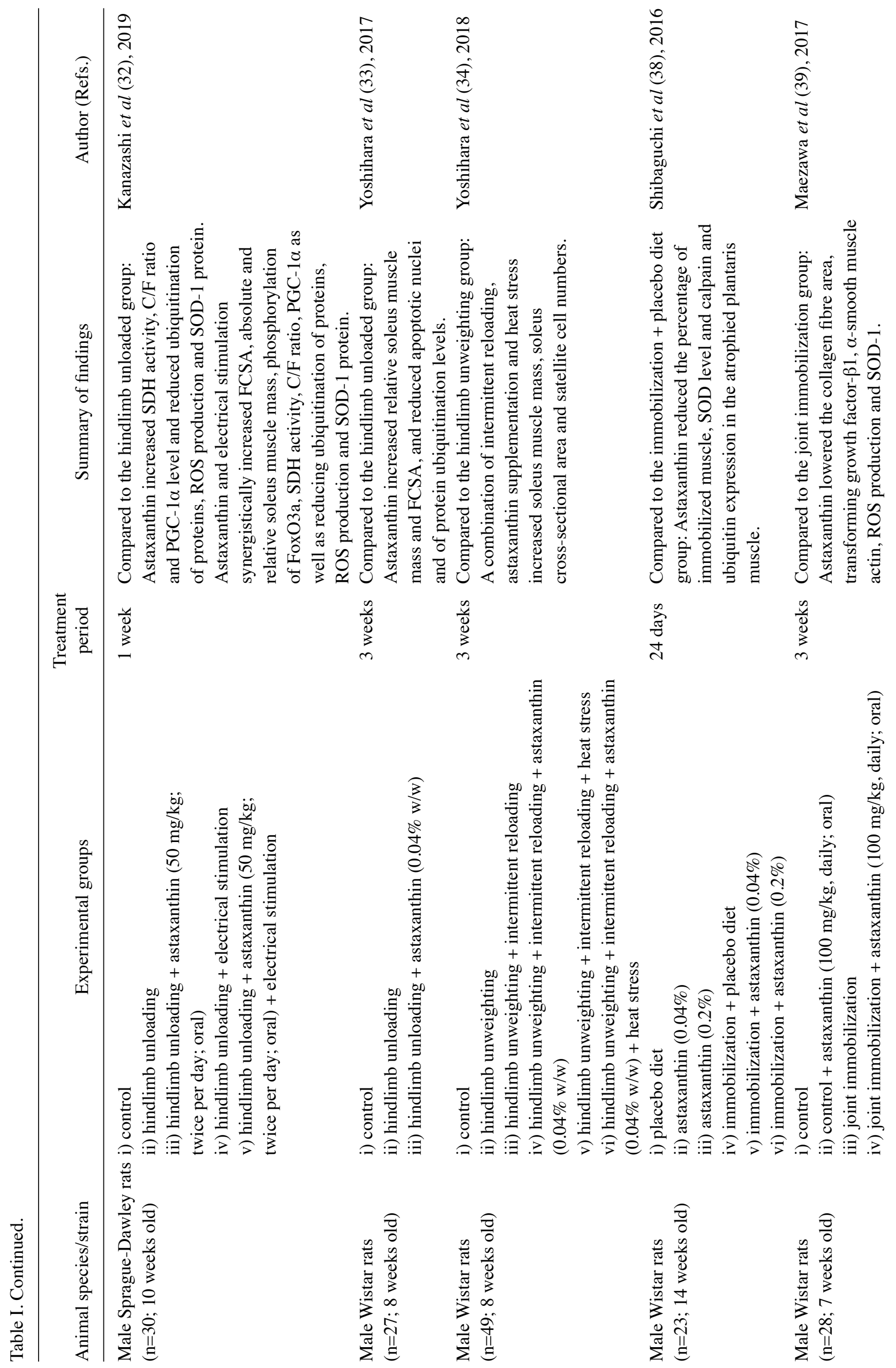




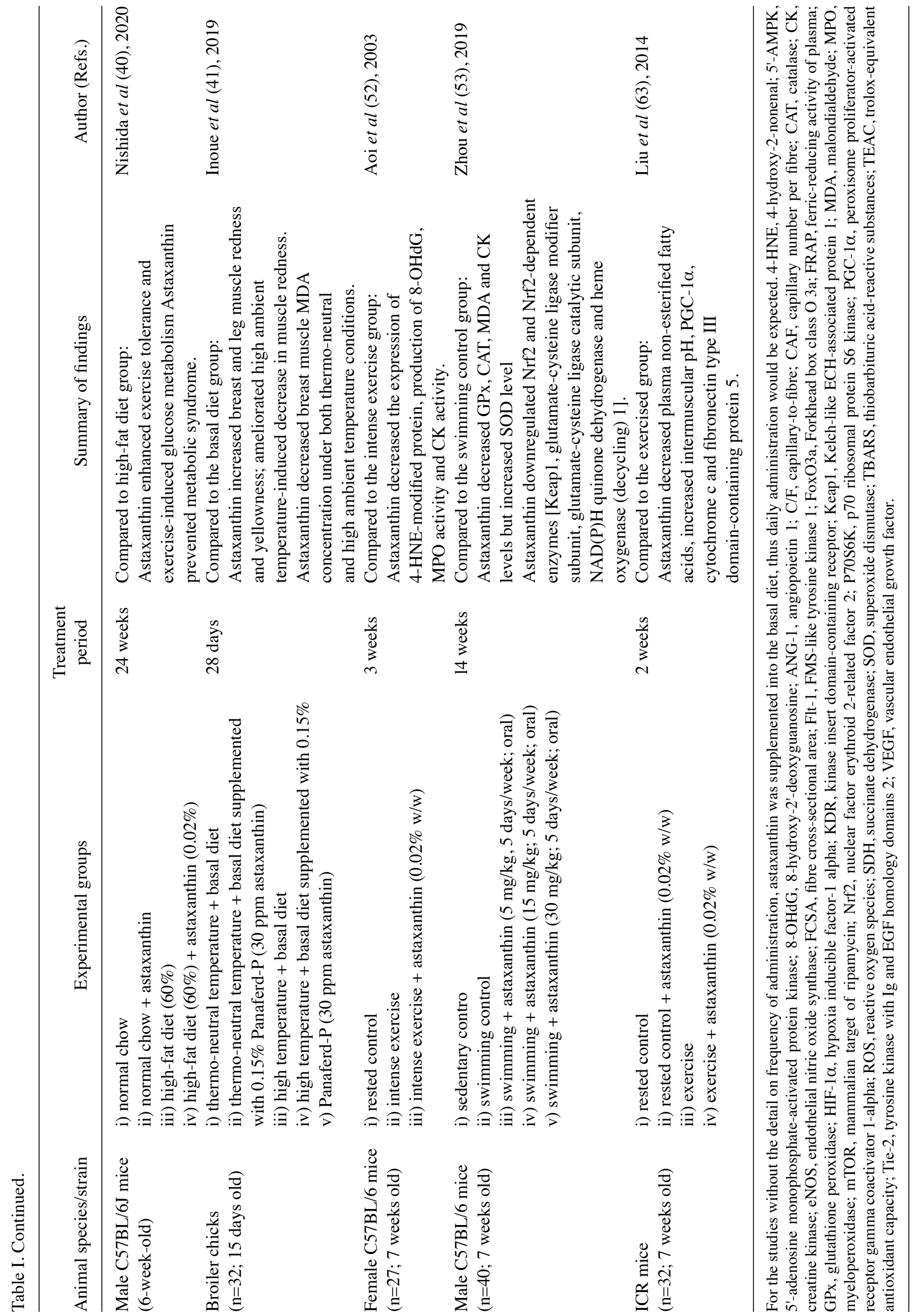


evaluate the effects of a combined therapy of astaxanthin and electrical stimulation on muscle atrophy using hindlimb unloaded rats. For electrical stimulation, calf muscles of the rats were electrically stimulated using a surface electrode (diameter: $1 \mathrm{~cm}$, frequency: $100 \mathrm{~Hz}$ ) for $240 \mathrm{sec} /$ day. Treatment with astaxanthin alone increased the $\mathrm{C} / \mathrm{F}$ ratio. The combined therapy was more efficient than astaxanthin alone in reversing the adverse changes due to hindlimb unloading. The combination of astaxanthin and electrical stimulation increased absolute soleus muscle mass and fibre cross-sectional area (FCSA) (32). Another group of researchers reported that dietary astaxanthin supplementation prior to and during hindlimb unloading suppressed soleus muscle atrophy. Compared to the animals subjected to hindlimb unloading without treatment, astaxanthin supplementation caused higher muscle weight and FCSA in the soleus muscle (33). A comprehensive study done by Yoshihara et al (34) illustrated the effects of a combination of astaxanthin supplementation, heat stress and intermittent reloading on the hindlimb unweighted rats. Hindlimb unloading was conducted as aforementioned, whereby the tail was immobilized in a cast, allowing the animals to move only using their front feet. The animals were placed in a heat chamber at $41.0-41.5^{\circ} \mathrm{C}$ for $30 \mathrm{~min}$. Intermittent reloading was performed during the heating phase for one hour every other day to allow daily activities. Astaxanthin was mixed into their basal diet at $0.04 \% \mathrm{w} / \mathrm{w}$. The combination of dietary astaxanthin, heat treatment and intermittent reloading resulted in higher soleus muscle weight and cross-sectional area in the hindlimb unloaded animals (34).

Hindlimb immobilization is another method to induce muscle atrophy. Immobilization refers to holding a joint or bone in place with a cast to prevent its movement, thus inducing muscle contracture and atrophy $(35,36)$. In contrast, the rodents are in a head-down position to simulate weightlessness for hindlimb unloading (37). In an in vivo study, three groups of male Wistar rats were given either a placebo diet, or a 0.04 or $0.2 \%$ astaxanthin diet for 24 days. At day 14 , hindlimb muscle immobilization was introduced to the rats in the maximum plantar flexion position with a plaster cast. It was demonstrated that the degree of muscle atrophy was lessened in the rats fed with a diet supplemented with astaxanthin (38). Similarly, Maezawa et al (39) introduced joint immobilization to 7-week-old male Wistar rats using the same approach. Astaxanthin $(100 \mathrm{mg} / \mathrm{kg})$ was administered orally each day for three weeks (one week before and two weeks during ankle joint immobilization). The treatment of astaxanthin reduced FCSA in the rats with joint immobilization (39).

In high-fat diet fed male C57BL/6J mice, astaxanthin was shown to increase exercise endurance. The astaxanthin-treated mice were able to run for a longer distance than the untreated mice when subjected to daily exercise using a treadmill and wheel. Astaxanthin also increased glucose tolerance after regular daily training, along with other metabolic syndrome parameters [fasting blood glucose, insulin, homeostatic model assessment of insulin resistance (HOMA-IR), glycated haemoglobin (HbA1c), systolic blood pressure, triglyceride and total cholesterol were reduced] (40).

The impact of astaxanthin supplementation on meat colouration in chickens has been evaluated. Using 15-day-old Broiler chicks as an animal model, Inoue et al (41) randomly assigned the chicks to one of the four groups using a $2 \times 2$ factorial design. The two main variables in this study were diet [basal diet or basal diet enriched with $0.15 \%$ Panaferd-P (containing $30 \mathrm{ppm}$ astaxanthin)] and ambient temperature [thermo-neutral temperature $\left(25 \pm 1^{\circ} \mathrm{C}\right)$ or high temperature $\left(35 \pm 1^{\circ} \mathrm{C}\right)$ ]. It was revealed that a diet containing Panaferd-P increased muscle carotenoid content, redness and yellowness of the skeletal muscle (meat) in the broiler chicks under the condition of thermo-neutral and high ambient temperature (41). Meat colour determines meat quality (42). A decrease in muscle redness might be a consequence of an alteration in muscle myoglobin concentration (the main protein responsible for meat colour), heat stress and feed restriction $(42,43)$. Meanwhile, a reduction in muscle yellowness is an indicator for decreasing carotenoid (astaxanthin, adonixanthin, canthaxanthin, adonirubin, lutein and zeaxanthin) accumulations in muscle. Hence, the increases in muscle redness and yellowness indicated quality improvement of the meat (41).

\section{Effects of astaxanthin on skeletal muscle: Evidence from human studies}

Limited studies have been conducted in humans to test the effects of astaxanthin on muscle, particularly in the aspects of muscle injury/damage and muscle strength (Table II). The effects of astaxanthin on muscle injury were studied among resistance-trained men $(n=20$, aged $25.1 \pm 1.6$ years). The subjects were equally divided into the placebo (administered $1,732 \mathrm{mg}$ safflower oil) or astaxanthin (administered $4 \mathrm{mg}$ astaxanthin and $480 \mathrm{mg}$ lutein) groups. After three weeks of assigned treatments, the participants were subjected to eccentric exercise (10 sets of 10 repetitions at $85 \%$ of one repetition maximum) and followed through $96 \mathrm{~h}$ post-exercise. The parameters measured in this study include muscle soreness, creatine kinase (CK) activity and muscle performance. A similar response in these variables was noted for both groups, reiterating that astaxanthin supplementation exerted negligible effects on skeletal muscle injury following eccentric loading (44). Another human study demonstrated the effects of astaxanthin supplementation (4 mg) for 90 days on muscle damage, oxidative stress and antioxidant capacity during soccer training in elite young soccer players. Treatment with astaxanthin did not change the levels of thiobarbituric acid-reactive substances (TBARS) and advanced oxidation protein products (AOPP) throughout this study. The $\mathrm{CK}$ and aspartate aminotransferase (AST) activities in serum were significantly increased with soccer training without treatment but were lowered with astaxanthin administration (45). In a randomized, double-blind, placebo-controlled study, Liu et al (46) examined a test formulation consisting of astaxanthin (12 mg), tocotrienol (10 mg) and zinc (6 mg) on building strength, endurance and mobility in exercise training among the elderly. A total of 42 elderly subjects (aged 65-85 years) were recruited, fed with test formulation or placebo for 4 months and trained with increasing intervals of incline walking for three months (three times weekly for 40-60 min). In this study, muscle strength was presented as maximal voluntary contraction (MVC) in an ankle dorsiflexion exercise, and the tibialis anterior muscle size was measured as cross-sectional area (CSA) using magnetic resonance imaging. The authors 


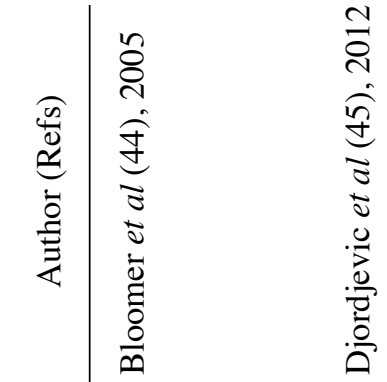

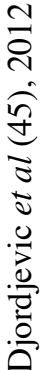

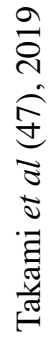

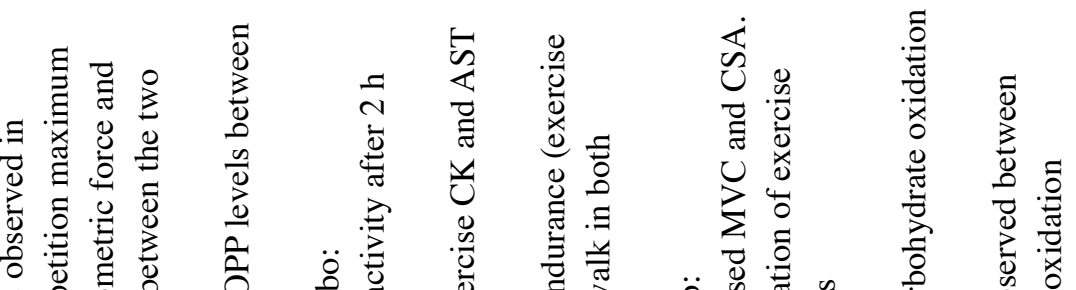

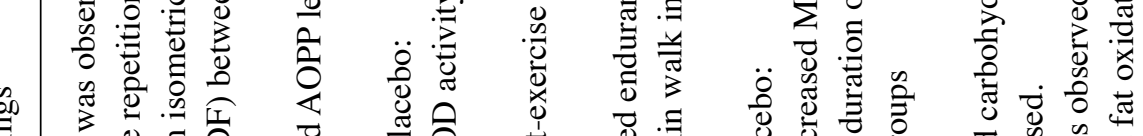

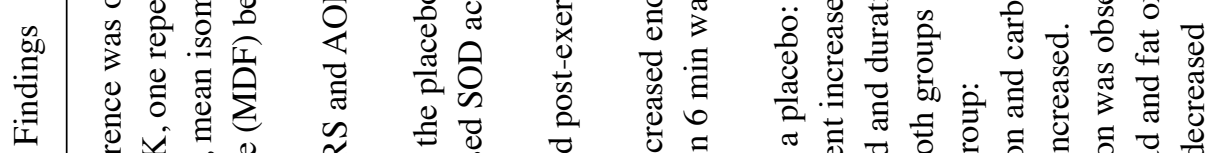

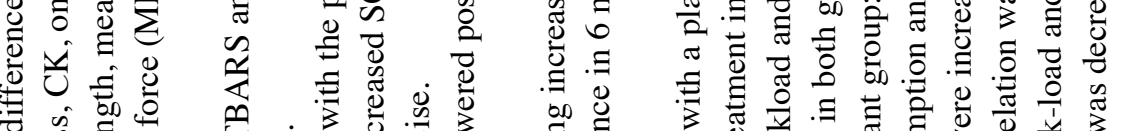

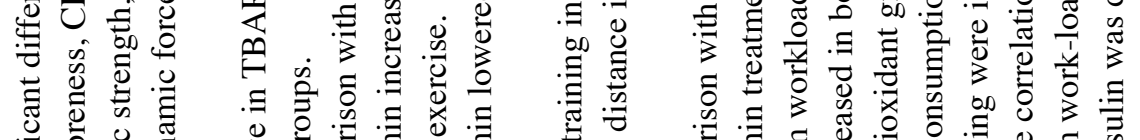

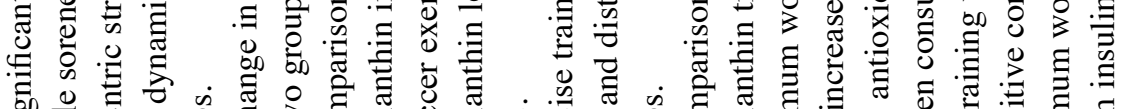

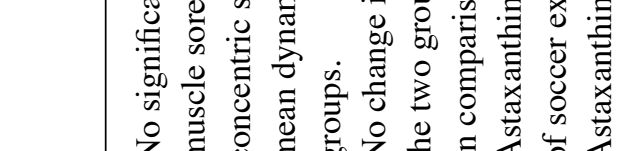

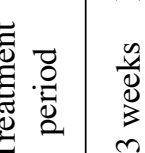
要

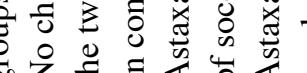

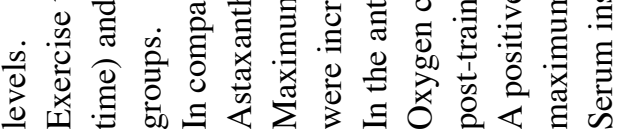

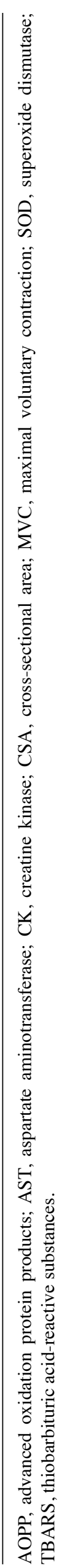


identified a greater endurance in a 6-min walk upon exercise training in both experimental groups. The subjects administered astaxanthin formulation had higher MVC and CSA, indicating improved muscle strength and size as compared to the placebo-treated exercised subjects (46).

A recent study by Takami et al (47) assessed whether foods containing antioxidants (such as catechin, astaxanthin, quercetin, glutathione and anthocyanin) could boost aerobic metabolism during exercise training. All participants were divided into two groups subjected to supervised cycling training for $30 \mathrm{~min}$ (three days per week) for four weeks with or without taking antioxidant-rich foods. Several observations were made in this study. The values of oxygen consumption and carbohydrate oxidation after training during rest and exercise conditions were significantly increased in the antioxidant group. A positive correlation was observed between fat oxidation during exercise and maximum workload after training. The magnitude of decrease in serum insulin level after training was higher in the antioxidant group as compared to the control group (47).

Overall, the evidence derived from in vivo studies suggested a beneficial effect of astaxanthin in preventing muscle degeneration. In humans, the effects of astaxanthin alone or in combination with other antioxidants on muscle health were heterogenous, as both positive and negligible effects were reported.

\section{The mechanism of action of astaxanthin}

Understanding of the biological mechanisms underlying the decline in muscle strength and mass is of substantial importance in the search for potential therapeutic agents to prevent sarcopenia. The widely accepted mechanisms involved in muscle atrophy leading to pathogenesis of sarcopenia include induction of oxidative stress, impaired mitochondrial dynamics and functions, negative protein turnover (defined as a disproportionate decrease in muscle protein synthesis and/or an increase in muscle protein breakdown) as well as regression of the capillary network in skeletal muscle (39).

Oxidative stress exerts dual actions on skeletal muscle, whereby a low level of oxidative stress is beneficial while excessive oxidative stress is detrimental (48). Oxidative stress is closely associated with sarcopenia, which is largely attributed to the excessive yield of reactive oxygen and nitrogen species (RONS) during ageing, high-intensity exercise and disuse atrophy (49). An increase in ROS level inflicts direct alteration or damage on important macromolecules, such as lipids, proteins and nucleic acids, contributing to the loss of muscle mass and strength (50). The anti-oxidative properties of astaxanthin have been widely demonstrated by researchers, evidenced by reduction in various lipid peroxidation by-products, oxidative stress biomarkers and markers of muscle damage (51). An earlier animal study demonstrated that astaxanthin attenuated exercise-induced skeletal and cardiac muscle damage in 7-week-old female C57BL/6 mice. The animals were randomly assigned to three groups: Rested controls, intense exercise and intense exercise supplemented with dietary astaxanthin $(0.02 \% \mathrm{w} / \mathrm{w})$. Exercise acclimation (running on a motor-driven treadmill with running intensity increased from 5 to $28 \mathrm{~m} / \mathrm{min}$ ) performed for $10 \mathrm{~min} /$ day three times per week for three weeks. At the end of the study, the exercise groups ran on a treadmill at $28 \mathrm{~m} / \mathrm{min}$ until exhaustion. The data from this study showed that increases in 4-hydroxy-2-nonenal (4-HNE)-modified protein, 8-hydroxy-2'-deoxyguanosine (8-OHdG), plasma CK activity and myeloperoxidase (MPO) activity in the gastrocnemius and heart caused by exercise were attenuated by astaxanthin (52). Astaxanthin treatment was effective in lowering the concentrations of malondialdehyde (MDA) or TBARS, ROS and carbonylated protein in various animal models $(28-32,38,39,41,53)$.

The complex endogenous antioxidant defence system, consisting of key antioxidant enzymes such as glutathione peroxidase (GPx), superoxide dismutase (SOD) and catalase (CAT), plays a crucial role in neutralizing damaging free radical species (54). High doses of astaxanthin ( 15 or $30 \mathrm{mg} / \mathrm{kg}$ ) were shown to be effective in suppressing the levels of GPx, CAT and CK and raising SOD activity in plasma and muscle of mice after moderate-intensity swimming training (53). Another group of researchers pinpointed the reduction in SOD-1 expression in animals with muscle atrophy induced by hindlimb unloading treated with astaxanthin alone (30) or in combination with other interventions such as intermittent loading (31) or electrical stimulation (32). In line with these studies, a lowered SOD-1 level was also detected in two other studies using animals subjected to hindlimb immobilization-induced muscle atrophy $(38,39)$. Astaxanthin supplementation also increased antioxidant capacity in the plasma, indicated by higher Trolox-equivalent antioxidant capacity (TEAC) levels and ferric-reducing activity of plasma (FRAP) capacity relative to the control animals (28).

At the molecular level, the signalling pathway involved in normalizing the disrupted balance between pro-oxidant and antioxidant levels is that of the nuclear factor erythroid 2-related factor 2 (Nrf2) (55). In the resting condition, Nrf2 assumes an inactive state by binding to Kelch-like ECH-associated protein 1 (Keapl) to cause its ubiquitination and degradation (56). Oxidative stress causes a conformational change in Keapl by interfering in the interaction between Nrf2 and Keap1. Free Nrf2 is subsequently released, translocated into the nucleus and bound to antioxidant responsive elements (ARE) to allow transcription of genes that encode for detoxifying or antioxidant enzymes (56). The transcription of Nrf2 and Nrf2-dependent enzymes in the mouse heart during moderate-intensity swimming training were downregulated in astaxanthin-treated animals (53). In this context, it appears that either the lack of or excess of ROS and antioxidants elicited important pathological implications in skeletal muscle. An optimal amount of ROS and antioxidants may serve as an important factor in maximizing skeletal muscle performance.

The mitochondrial electron transport chain is the major site of ROS production in skeletal muscle, thus mitochondrial DNA is susceptible to oxidative damage by overwhelming ROS production, affecting mitochondrial homeostasis and function (57). Changes in mitochondrial membrane potential, reduction in mitochondrial energy production capacity, inhibition of mitochondrial oxygen consumption and reduction in mitochondrial biogenesis are common characteristics of mitochondrial dysfunction (58), which are (59). During physical activity, endothelial nitric associated with the development of sarcopenia oxide synthase (eNOS) is upregulated 


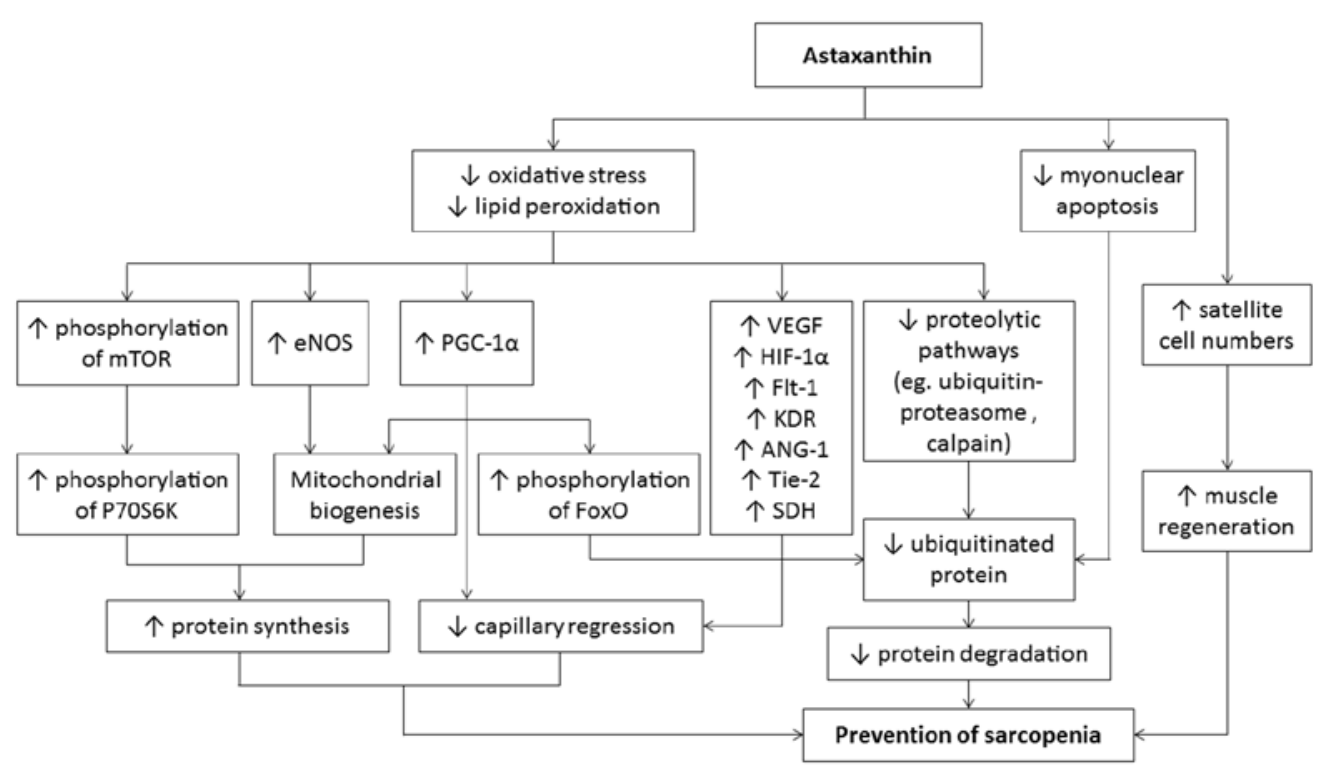

Figure 1. The potential mechanism of action of astaxanthin in the prevention of sarcopenia. ANG-1, angiopoietin 1; eNOS, endothelial nitric oxide synthase; Flt-1, FMS-like tyrosine kinase 1; FoxO, Forkhead Box O; HIF-1 $\alpha$, hypoxia inducible factor-1 alpha; KDR, kinase insert domain-containing receptor; mTOR, mammalian target of ripamycin; P70S6K, p70 ribosomal protein S6 kinase; PGC-1 $\alpha$, peroxisome proliferator-activated receptor gamma coactivator 1-alpha; SDH, succinate dehydrogenase; Tie-2, tyrosine kinase with Ig and EGF homology domains 2; VEGF, vascular endothelial growth factor.

to increase nitric oxide production, which subsequently induces mitochondrial biogenesis and cell glucose uptake in skeletal muscle. Peroxisome proliferator-activated receptor gamma coactivator $1-\alpha(\mathrm{PGC}-1 \alpha)$ is a master regulator of mitochondrial biogenesis that regulates the genes involved in cellular energy metabolism $(60,61)$. High levels of PGC- $1 \alpha$ are an indicator of improved aerobic metabolism and function of mitochondria (62). Using an exercised mouse model, Liu et al (63) suggested that astaxanthin accelerated lipid utilization in skeletal muscle and reduced intermuscular $\mathrm{pH}$ during aerobic exercise through elevation of PGC-1 $\alpha$. Studies conducted by Kanazashi et al $(31,32)$ identified a similar pattern to astaxanthin alone, astaxanthin with intermittent loading and astaxanthin with electrical stimulation retained mitochondrial biogenesis by raising PGC- $1 \alpha$ and eNOS expression in the soleus muscle of hindlimb unloaded mice. The total 5'-adenosine monophosphate-activated protein kinase (AMPK) content in skeletal muscle was also significantly augmented in the exercised animals fed with astaxanthin from $H$. pluvialis than the control group. These findings suggested that astaxanthin enhanced energy production leading to a longer running time during treadmill exercise (27).

Under physiological conditions, the maintenance of skeletal muscle mass depends on the balance between muscle protein synthesis and muscle protein degradation (64). Muscle atrophy occurs when the rate of protein degradation outweighs the rate of protein synthesis (65). The suggested signal transduction involved in muscle protein synthesis and degradation includes the phosphatidylinositol-3-kinase $(\mathrm{PI} 3 \mathrm{~K})$ /protein kinase $\mathrm{B}$ (Akt)/mammalian target of ripamycin (mTOR) signalling and Forkhead Box O (FoxO) transcription factor. The activation of $\mathrm{PI} 3 \mathrm{~K} / \mathrm{Akt} / \mathrm{mTOR}$ pathway is modulated by the interaction of insulin growth factor-1 (IGF-1) and insulin with their respective tyrosine kinase receptors. The activated PI3K/Akt eventually phosphorylates mTOR and its downstream factor, p70 ribosomal protein S6 kinase (P70S6K) to promote protein synthesis $(66,67)$. The mixture of three antioxidants (astaxanthin, $\beta$-carotene and resveratrol) was shown to have greater efficacy than each antioxidant respectively in increasing relative soleus weight. This outcome was mediated through the increased phosphorylation of mTOR and its downstream factor (P70S6K) in male mice with muscle atrophy (29). FoxO transcription factors play a role in the catabolic pathway in skeletal muscle. FoxO is phosphorylated (inhibited) by Akt, thus the genes responsible for muscle atrophy cannot be transcribed (68). In the study performed by Kanazashi et al (32), it was noted that hindlimb unloading induced muscle atrophy in the rats by activating the ubiquitin-proteasome pathway through reduced phosphorylation (activation) of Forkhead box class $\mathrm{O} 3 \mathrm{a}$ (FoxO3a). It is also evident that protein degradation during muscle disuse is associated with activation of the ubiquitin-proteasome proteolytic pathway, resulting in increased ubiquitinated protein expression. Muscle atrophy induced by hindlimb unloading was reversed by the combined intervention of astaxanthin and electrical stimulation via increased phosphorylation (inhibition) of FoxO3a (32). In addition, it has been reported that the induction of oxidative stress stimulated protein degradation by upregulating calpain (a proteolytic enzyme act upstream of the ubiquitin-proteasome proteolytic pathway) (69). It was revealed that dietary astaxanthin intake protected against disuse muscle atrophy in rats, which was partly due to the reduction of oxidative stress, calpain and ubiquitin expression (38).

Another mechanism of action that explains the positive effects of astaxanthin in suppressing disuse skeletal muscle atrophy involves the inhibition of myonuclear apoptosis. Apoptosis of myonuclei contributes to the loss of muscle mass. Previous work by Yoshihara et al (33) indicated that dietary astaxanthin supplementation prevented the increase of apoptotic nuclei in soleus muscle [indicated by decreased 
number of terminal deoxynucleotidyl transferase dUTP nick end labelling (TUNEL)-positive nuclei]. Satellite cells (also known as skeletal muscle stem cells) are the precursors of skeletal muscle cells required for muscle mass maintenance and muscle regeneration following muscle atrophy (70). Previous studies have demonstrated the alterations of satellite cell activity and density by muscle catabolic conditions, such as disuse and ageing (71). Indeed, Yoshihara and co-authors (34) also revealed that the protection against disuse muscle atrophy exerted by astaxanthin might be due to the increase in satellite cell numbers.

The capillary number in skeletal muscle is proportionate with muscle loading and activity levels. For instance, exercise and functional overload promote capillary growth (72) whereas unloading and immobilization result in capillary regression (73). The regression of the capillary network during low level muscle loading and activity is often attributed to an increase in oxidative injury (11). Kanazashi et al $(30,31)$ performed two studies to assess the effects of astaxanthin on skeletal muscle capillaries. In these studies, they reported that hindlimb unloading induced an overproduction of ROS, resulting in capillary regression and muscle atrophy in the animals. Upon astaxanthin intervention, the decreases in angiogenic factors [such as vascular endothelial growth factor (VEGF), hypoxia inducible factor-1 alpha (HIF-1 $\alpha$ ), FMS-like tyrosine kinase 1 (Flt-1), kinase insert domain-containing receptor (KDR), angiopoietin 1 (ANG-1) and tyrosine kinase with Ig and EGF homology domains 2 (Tie-2)] caused by hindlimb unloading were counteracted (30). The subsequent study revealed that hindlimb unloading decreased PGC-1 $\alpha$, VEGF and succinate dehydrogenase (SDH) activity, which contributed to the detrimental effects on morphology and number of capillary networks in rat soleus muscle. Oral astaxanthin administration maintained the capillary network by increasing PGC-1 $\alpha$, VEGF and SDH activity near values of animals with sarcopenia (31).

\section{Perspectives and conclusion}

In the present review, the role of astaxanthin on skeletal muscle was examined in two major conditions: Physical exercise and muscle atrophy. Though the direct beneficial effects of astaxanthin on skeletal muscle were marginal in certain studies, astaxanthin was shown to be a potentially effective agent to enhance skeletal muscle performance and counteract the detrimental effects of skeletal muscle disuse. The mechanisms of action of astaxanthin may be attributed to its potential to prevent oxidative stress, increase energy production in mitochondria, regulate the anabolic (regeneration) and catabolic (proteolysis) processes of skeletal muscle, suppress programmed cell death of the myonucleus and activate associated angiogenic pathways to maximize capillary network (Fig. 1). Among these molecular mechanisms, oxidative stress appears to be the common factor that ultimately causes stepwise escalation to the onset and progression of sarcopenia.

Several limitations of the currently available studies need to be addressed. Firstly, the evidence is largely preliminary and suggestive of the potential of astaxanthin in the management of sarcopenia. Much effort should be paid on further investigations to validate the clinical use of astaxanthin. Secondly, the induction of oxidative stress in skeletal muscle has a direct mechanistic link with chronic state of low-grade inflammation during disuse muscle atrophy (50). Despite exhibiting anti-oxidative properties, astaxanthin has been reported to be useful for improving chronic inflammation $(24,74)$. Therefore, investigation of the anti-inflammatory properties of astaxanthin during exercise or skeletal muscle atrophy may be a beneficial area of research. Thirdly, the test formulation provided in certain studies was a mixture of astaxanthin with other antioxidants. The positive health outcomes of astaxanthin alone could not be concluded as the effects might be derived from other antioxidative agents. As a combination of astaxanthin with other interventions may show greater efficacy than astaxanthin alone in promoting skeletal muscle health and performance astaxanthin may be beneficial when used clinically in conjunction with other interventions, such as exercise, hormonal and nutritional intervention to improve muscle health.

\section{Acknowledgements}

Not applicable.

\section{Funding}

This study was supported by grants from the Universiti Kebangsaan Malaysia and Ministry of Education, Malaysia (grant nos. MI-2019-006 and FRGS/1/2018/SKK10/UKM/03/1).

\section{Availability of data and materials}

Not applicable.

\section{Authors' contributions}

SKW performed the literature search and drafted the manuscript. SIN and KYC provided critical review for the manuscript. KYC gave final approval for the publication of this manuscript. All authors read and approved the final manuscript.

\section{Ethics approval and consent to participate}

Not applicable.

\section{Patient consent for publication}

Not applicable.

\section{Competing interests}

The authors declare that they have no competing interests.

\section{References}

1. Santilli V, Bernetti A, Mangone M and Paoloni M: Clinical definition of sarcopenia. Clin Cases Miner Bone Metab 11: 177-180, 2014.

2. Trajanoska K, Schoufour JD, Darweesh SK, Benz E, Medina-Gomez C, Alferink LJ, Lahousse L, Brusselle G, Stricker B, Darwish Murad S, et al: Sarcopenia and its clinical correlates in the general population: The rotterdam study. J Bone Miner Res 33: 1209-1218, 2018. 
3. Beaudart C, Rizzoli R, Bruyère $\mathrm{O}$, Reginster JY and Biver E: Sarcopenia: Burden and challenges for public health. Arch Public Health 72: 45-45, 2014.

4. Shafiee G, Keshtkar A, Soltani A, Ahadi Z, Larijani B and Heshmat R: Prevalence of sarcopenia in the world: A systematic review and meta-analysis of general population studies J Diabetes Metab Disord 16: 21-21, 2017.

5. Goates S, Du K, Arensberg MB, Gaillard T, Guralnik J and Pereira SL: Economic impact of hospitalizations in US adults with sarcopenia. J Frailty Aging 8: 93-99, 2019.

6. World Population Ageing: World Population Ageing 2017. United Nations New York, 2017.

7. Kwak JY and Kwon KS: Pharmacological interventions for treatment of sarcopenia: Current status of drug development for sarcopenia. Ann Geriatr Med Res 23: 98-104, 2019.

8. Aoi W and Sakuma K: Oxidative stress and skeletal muscle dysfunction with aging. Curr Aging Sci 4: 101-109, 2011.

9. Londhe P and Guttridge DC: Inflammation induced loss of skeletal muscle. Bone 80: 131-142, 2015.

10. Andreux PA, van Diemen MPJ, Heezen MR, Auwerx J, Rinsch C, Groeneveld GJ and Singh A: Mitochondrial function is impaired in the skeletal muscle of pre-frail elderly. Sci Rep 8: 8548,2018

11. Fujino H, Kondo H, Nagatomo F and Ishihara A: Capillary growth and regression in skeletal muscle. J Phys Fit Sports Med 3: 483-491, 2014

12. Lee SP, Mar GY and Ng LT: Effects of tocotrienol-rich fraction on exercise endurance capacity and oxidative stress in forced swimming rats. Eur J Appl Physiol 107: 587-595, 2009.

13. Servais S, Letexier D, Favier R, Duchamp C and Desplanches D: Prevention of unloading-induced atrophy by vitamin E supplementation: Links between oxidative stress and soleus muscle proteolysis? Free Radic Biol Med 42: 627-635, 2007.

14. Higuera-Ciapara I, Felix-Valenzuela L and Goycoolea FM: Astaxanthin: A review of its chemistry and applications. Crit Rev Food Sci Nutr 46: 185-196, 2006.

15. Dose J, Matsugo S, Yokokawa H, Koshida Y, Okazaki S, Seidel U, Eggersdorfer M, Rimbach G and Esatbeyoglu T: Free radical scavenging and cellular antioxidant properties of astaxanthin. Int J Mol Sci 17: 103, 2016.

16. Park JS, Chyun JH, Kim YK, Line LL and Chew BP: Astaxanthin decreased oxidative stress and inflammation and enhanced immune response in humans. Nutr Metab 7: 18-18, 2010.

17. McCall B, McPartland CK, Moore R, Frank-Kamenetskii A and Booth BW: Effects of astaxanthin on the proliferation and migration of breast cancer cells in vitro. Antioxidants 7: 135, 2018

18. Sila A, Ghlissi Z, Kamoun Z, Makni M, Nasri M, Bougatef A and Sahnoun Z: Astaxanthin from shrimp by-products ameliorates nephropathy in diabetic rats. Eur J Nutr 54: 301-307, 2015.

19. Kang $\mathrm{H}$ and $\mathrm{Kim} \mathrm{H}$ : Astaxanthin and $\beta$-carotene in Helicobacter pylori-induced Gastric Inflammation: A mini-review on action mechanisms. J Cancer Prev 22: 57-61, 2017.

20. Rao AR, Sarada R, Shylaja MD and Ravishankar GA: Evaluation of hepatoprotective and antioxidant activity of astaxanthin and astaxanthin esters from microalga-Haematococcus pluvialis. J Food Sci Tech 52: 6703-6710, 2015.

21. Feng Y, Chu A, Luo Q, Wu M, Shi X and Chen Y: The protective effect of astaxanthin on cognitive function via inhibition of oxidative stress and inflammation in the brains of chronic T2DM Rats. Front Pharmacol 9: 748, 2018.

22. Fassett RG and Coombes JS: Astaxanthin: A potential therapeutic agent in cardiovascular disease. Mar Drugs 9: 447-465, 2011.

23. Hashimoto H, Arai K, Hayashi S, Okamoto H, Takahashi J, Chikuda $\mathrm{M}$ and Obara Y: Effects of astaxanthin on antioxidation in human aqueous humor. J Clin Biochem Nutr 53: 1-7, 2013.

24. Davinelli S, Nielsen ME and Scapagnini G: Astaxanthin in skin health, repair, and disease: A comprehensive review. Nutrients 10: $522,2018$.

25. Brown DR, Gough LA, Deb SK, Sparks SA and McNaughton LR: Astaxanthin in exercise metabolism, performance and recovery: A review. Front Nutr 4: 76, 2017

26. Yu T, Dohl J, Chen Y, Gasier HG and Deuster PA: Astaxanthin but not quercetin preserves mitochondrial integrity and function, ameliorates oxidative stress, and reduces heat-induced skeletal muscle injury. J Cell Physiol 234: 13292-13302, 2019.

27. Aoi W, Maoka T, Abe R, Fujishita $M$ and Tominaga $K$ : Comparison of the effect of non-esterified and esterified astaxanthins on endurance performance in mice. J Clin Biochem Nutr 62: 161-166, 2018.
28. Polotow TG, Vardaris CV, Mihaliuc AR, Gonçalves MS, Pereira B, Ganini D and Barros MP: Astaxanthin supplementation delays physical exhaustion and prevents redox imbalances in plasma and soleus muscles of Wistar rats. Nutrients 6: 5819-5838, 2014.

29. Kawamura A, Aoi W, Abe R, Kobayashi Y, Wada S, Kuwahata M and Higashi A: Combined intake of astaxanthin, $\beta$-carotene, and resveratrol elevates protein synthesis during muscle hypertrophy in mice. Nutrition 69: 110561, 2019.

30. Kanazashi M, Okumura Y, Al-Nassan S, Murakami S, Kondo H, Nagatomo F, Fujita N, Ishihara A, Roy RR and Fujino H: Protective effects of astaxanthin on capillary regression in atrophied soleus muscle of rats. Acta Physiol 207: 405-415, 2013.

31. Kanazashi M, Tanaka M, Murakami S, Kondo H, Nagatomo F, Ishihara A, Roy RR and Fujino H: Amelioration of capillary regression and atrophy of the soleus muscle in hindlimb-unloaded rats by astaxanthin supplementation and intermittent loading. Exp Physiol 99: 1065-1077, 2014

32. Kanazashi M, Tanaka M, Nakanishi R, Maeshige $\mathrm{N}$ and Fujino H: Effects of astaxanthin supplementation and electrical stimulation on muscle atrophy and decreased oxidative capacity in soleus muscle during hindlimb unloading in rats. J Physiol Sci 69: 757-767, 2019.

33. Yoshihara T, Yamamoto Y, Shibaguchi T, Miyaji N, Kakigi R, Naito H, Goto K, Ohmori D, Yoshioka T and Sugiura T: Dietary astaxanthin supplementation attenuates disuse-induced muscle atrophy and myonuclear apoptosis in the rat soleus muscle. J Physiol Sci 67: 181-190, 2017.

34. Yoshihara T, Sugiura T, Miyaji N, Yamamoto Y, Shibaguchi T, Kakigi R, Naito H, Goto K, Ohmori D and Yoshioka T: Effect of a combination of astaxanthin supplementation, heat stress, and intermittent reloading on satellite cells during disuse muscle atrophy. J Zhejiang Univ Sci B 19: 844-852, 2018.

35. Wang F, Zhang QB, Zhou Y, Chen S, Huang PP, Liu Y and $\mathrm{Xu}$ YH: The mechanisms and treatments of muscular pathological changes in immobilization-induced joint contracture: A literature review. Chin J Traumatol 22: 93-98, 2019.

36. Frimel TN, Kapadia F, Gaidosh GS, Li Y, Walter GA and Vandenborne K: A model of muscle atrophy using cast immobilization in mice. Muscle Nerve 32: 672-674, 2005.

37. Morey-Holton ER and Globus RK: Hindlimb unloading rodent model: Technical aspects. J Appl Physiol (1985) 92: 1367-1377, 2002

38. Shibaguchi T, Yamaguchi Y, Miyaji N, Yoshihara T, Naito H, Goto K, Ohmori D, Yoshioka T and Sugiura T: Astaxanthin intake attenuates muscle atrophy caused by immobilization in rats. Physiol Rep 4: e12885, 2016.

39. Maezawa T, Tanaka M, Kanazashi M, Maeshige N, Kondo H, Ishihara A and Fujino H: Astaxanthin supplementation attenuates immobilization-induced skeletal muscle fibrosis via suppression of oxidative stress. J Physiol Sci 67: 603-611, 2017.

40. Nishida Y, Nawaz A, Kado T, Takikawa A, Igarashi Y, Onogi Y, Wada T, Sasaoka T, Yamamoto S, Sasahara M, et al: Astaxanthin stimulates mitochondrial biogenesis in insulin resistant muscle via activation of AMPK pathway. J Cachexia Sarcopenia Muscle 11: 241-258, 2020

41. Inoue H, Shimamoto S, Takahashi H, Kawashima Y, Wataru S, Ijiri D and Ohtsuka A: Effects of astaxanthin-rich dried cell powder from Paracoccus carotinifaciens on carotenoid composition and lipid peroxidation in skeletal muscle of broiler chickens under thermo-neutral or realistic high temperature conditions. Anim Sci J 90: 229-236, 2019.

42. Mancini RA and Hunt MC: Current research in meat color. Meat Sci 71: 100-121, 2005.

43. Zeferino CP, Komiyama CM, Pelícia VC, Fascina VB, Aoyagi MM, Coutinho LL, Sartori JR and Moura AS: Carcass and meat quality traits of chickens fed diets concurrently supplemented with vitamins $\mathrm{C}$ and $\mathrm{E}$ under constant heat stress. Animal 10: 163-171, 2016

44. Bloomer RJ, Fry A, Schilling B, Chiu L, Hori N and Weiss L: Astaxanthin supplementation does not attenuate muscle injury following eccentric exercise in resistance-trained men. Int J Sport Nutr Exerc Metab 15: 401-412, 2005.

45. Djordjevic B, Baralic I, Kotur-Stevuljevic J, Stefanovic A, Ivanisevic J, Radivojevic N, Andjelkovic M and Dikic N: Effect of astaxanthin supplementation on muscle damage and oxidative stress markers in elite young soccer players. J Sports Med Phys Fitness 52: 382-392, 2012

46. Liu SZ, Ali AS, Campbell MD, Kilroy K, Shankland EG, Roshanravan B, Marcinek DJ and Conley KE: Building strength, endurance, and mobility using an astaxanthin formulation with functional training in elderly. J Cachexia Sarcopenia Muscle 9: 826-833, 2018 
47. Takami M, Aoi W, Terajima H, Tanimura Y, Wada S and Higashi A: Effect of dietary antioxidant-rich foods combined with aerobic training on energy metabolism in healthy young men. J Clin Biochem Nutr 64: 79-85, 2019.

48. Scicchitano BM, Pelosi L, Sica G and Musarò A: The physiopathologic role of oxidative stress in skeletal muscle. Mech Ageing Dev 170: 37-44, 2018.

49. Brioche $\mathrm{T}$ and Lemoine-Morel S: Oxidative stress, sarcopenia, antioxidant strategies and exercise: Molecular aspects. Curr Pharm Des 22: 2664-2678, 2016.

50. Meng SJ and Yu LJ: Oxidative stress, molecular inflammation and sarcopenia. Int J Mol Sci 11: 1509-1526, 2010.

51. Sztretye M, Dienes B, Gönczi M, Czirják T, Csernoch L, Dux L, Szentesi P and Keller-Pintér A: Astaxanthin: A potential mitochondrial-targeted antioxidant treatment in diseases and with aging. Oxid Med Cell Longev 3849692, 2019.

52. Aoi W, Naito Y, Sakuma K, Kuchide M, Tokuda H, Maoka T, Toyokuni S, Oka S, Yasuhara M and Yoshikawa T: Astaxanthin limits exercise-induced skeletal and cardiac muscle damage in mice. Antioxid Redox Signal 5: 139-144, 2003.

53. Zhou Y, Baker JS, Chen X, Wang Y, Chen H, Davison GW and Yan X: High-dose astaxanthin supplementation suppresses antioxidant enzyme activity during moderate-intensity swimming training in mice. Nutrients 11, 1244, 2019.

54. Kurutas EB: The importance of antioxidants which play the role in cellular response against oxidative/nitrosative stress: Current state. Nutr J 15: 71-71, 2016.

55. Vomund S, Schäfer A, Parnham MJ, Brüne B and von Knethen A: Nrf2, the master regulator of anti-oxidative responses. Int J Mol Sci 18: 2772, 2017

56. Bellezza I, Giambanco I, Minelli A and Donato R: Nrf2-Keap1 signaling in oxidative and reductive stress. Biochim Biophys Acta Mol Cell Res 1865: 721-733, 2018.

57. Guo C, Sun L, Chen X and Zhang D: Oxidative stress, mitochondrial damage and neurodegenerative diseases. Neural Regen Res 8: 2003-2014, 2013.

58. Ren J, Pulakat L, Whaley-Connell A and Sowers JR: Mitochondrial biogenesis in the metabolic syndrome and cardiovascular disease. J Mol Med 88: 993-1001, 2010.

59. Coen PM, Musci RV, Hinkley JM and Miller BF: Mitochondria as a target for mitigating Sarcopenia. Front Physiol 9: 1883, 2018

60. Liang H and Ward WF: PGC-1alpha: A key regulator of energy metabolism. Adv Physiol Educ 30: 145-151, 2006.

61. Litvinova L, Atochin DN, Fattakhov N, Vasilenko M, Zatolokin P and Kirienkova E: Nitric oxide and mitochondria in metabolic syndrome. Front Physiol 6: 20, 2015.

62. Austin S and St-Pierre J: PGC1 alpha and mitochondrial metabolism-emerging concepts and relevance in ageing and neurodegenerative disorders. J Cell Sci 125: 4963-4971, 2012 .
63. Liu PH, Aoi W, Takami M, Terajima H, Tanimura Y, Naito Y, Itoh Y and Yoshikawa T: The astaxanthin-induced improvement in lipid metabolism during exercise is mediated by a PGC-1 $\alpha$ increase in skeletal muscle. J Clin Biochem Nutr 54: 86-89, 2014.

64. Gordon BS, Kelleher AR and Kimball SR: Regulation of muscle protein synthesis and the effects of catabolic states. Int J Biochem Cell Biol 45: 2147-2157, 2013.

65. Sandri M: Protein breakdown in muscle wasting: Role of autophagy-lysosome and ubiquitin-proteasome. Int J Biochem Cell Biol 45: 2121-2129, 2013

66. Latres E, Amini AR, Amini AA, Griffiths J, Martin FJ, Wei Y, Lin HC, Yancopoulos GD and Glass DJ: Insulin-like growth factor-1 (IGF-1) inversely regulates atrophy-induced genes via the phosphatidylinositol 3-kinase/Akt/mammalian target of rapamycin (PI3K/Akt/mTOR) pathway. J Biol Chem 280: 2737-2744, 2005

67. Ziaaldini MM, Marzetti E, Picca A and Murlasits Z: Biochemical pathways of sarcopenia and their modulation by physical exercise: A narrative review. Front Med 4: 167, 2017.

68. Sanchez AM, Candau RB and Bernardi H: FoxO transcription factors: Their roles in the maintenance of skeletal muscle homeostasis. Cell Mol Life Sci 71: 1657-1671, 2014.

69. Smuder AJ, Kavazis AN, Hudson MB, Nelson WB and Powers SK: Oxidation enhances myofibrillar protein degradation via calpain and caspase-3. Free Radic Biol Med 49: 1152-1160, 2010.

70. Dumont NA, Bentzinger CF, Sincennes MC and Rudnicki MA: Satellite cells and skeletal muscle regeneration. Compr Physiol 5: 1027-1059, 2015

71. McKenna CF and Fry CS: Altered satellite cell dynamics accompany skeletal muscle atrophy during chronic illness, disuse, and aging. Curr Opin Clin Nutr Metab Care 20: 447-452, 2017.

72. Hoier B and Hellsten Y: Exercise-induced capillary growth in human skeletal muscle and the dynamics of VEGF. Microcirculation 21: 301-314, 2014.

73. Fujino H, Kohzuki H, Takeda I, Kiyooka T, Miyasaka T, Mohri S, Shimizu J and Kajiya F: Regression of capillary network in atrophied soleus muscle induced by hindlimb unweighting. J Appl Physiol 98: 1407-1413, 2005.

74. Miyachi M, Matsuno T, Asano K and Mataga I: Anti-inflammatory effects of astaxanthin in the human gingival keratinocyte line NDUSD-1. J Clin Biochem Nutr 56: 171-178, 2015.

This work is licensed under a Creative Commons Attribution-NonCommercial-NoDerivatives 4.0 International (CC BY-NC-ND 4.0) License. 